\title{
Residual Cardiovascular Risk-Is Inflammation the Primary Cause?
}

\author{
David S. Schade*, R. Philip Eaton
}

Division of Endocrinology and Metabolism, Department of Internal Medicine, School of Medicine, University of New Mexico, Albuquerque, NM, USA

Email: *dschade@salud.unm.edu

How to cite this paper: Schade, D.S. and Eaton, R.P. (2018) Residual Cardiovascular Risk-Is Inflammation the Primary Cause? World Journal of Cardiovascular Diseases, 8, 59-69.

https://doi.org/10.4236/wjcd.2018.81007

Received: December 19, 2017

Accepted: January 21, 2018

Published: January 24, 2018

Copyright $\odot 2018$ by authors and Scientific Research Publishing Inc. This work is licensed under the Creative Commons Attribution International License (CC BY 4.0).

http://creativecommons.org/licenses/by/4.0/

\begin{abstract}
Every cardiovascular clinical trial that has examined the beneficial effects of lowering LDL cholesterol to prevent cardiovascular events has demonstrated residual cardiovascular risk in the interventional treatment group. Residual risk is the term applied to the cardiovascular events (e.g., myocardial infarction, stroke, and cardiovascular death) that occur in spite of being on "optimal" medical therapy. This term is usually applied to secondary intervention studies, i.e., lipid lowering treatments in subjects who have already had at least one cardiovascular event. Studies that described residual risk have attributed it, at least in part, to the fact that the LDLc has not been lowered sufficiently to stop atherosclerotic plaque formation and rupture into the arterial lumen. However, a recent cardiovascular intervention clinical trial which achieved a very low group median LDLc of $30 \mathrm{mg} / \mathrm{dl}$ still demonstrated significant residual risk. Of more importance to reducing residual risk may be addressing the ongoing inflammation in the coronary arteries that results in cellular liberation of cytokines and proteases that attack the atherosclerotic plaque's fibrous cap. Recent studies have shown that inflammation may act independently of LDL to cause cardiovascular events. This article provides evidence that inflammation is the primary cause of residual risk and will need to be treated as aggressively as LDL lowering if CVD events in the post treatment period are to be significantly reduced. Addressing major risk factors including obesity, diabetes, smoking, hypertension and hyperlipidemia are critical to reducing inflammation. Statins and aspirin are the mainstay medications to reduce ongoing inflammation. However, newer pharmaceuticals may also be required to reduce inflammation to undetectable levels. Targeting inflammation to eradicate residual cardiovascular risk will be the next therapeutic challenge facing primary care physicians.
\end{abstract}

\section{Keywords}

Residual Risk, Inflammation, Cholesterol, C-Reactive Protein, Cardiovascular Events 


\section{Introduction}

Inflammation is a characteristic of all chronic diseases in man. It is the primary defense mechanism against invading organisms and hostile environmental agents. It has developed over millions of years of evolution and involves a multiplicity of genes, organ systems, cellular organelles, and active metabolites. When inflammation is active, it may be lifesaving to the individual. However, it also can cause great harm with damage to blood vessels, organs and the functional capacity of the organism. Atherosclerosis is a chronic disease characterized by increased inflammation affecting all of the arteries in the body. As a consequence, the endothelial lining of the arteries becomes damaged, resulting in the development of atherosclerotic plaques that lead to arterial thrombosis. Controlling this inflammatory response is critical to reducing atherosclerotic cardiovascular events.

Cardiovascular disease is the number one cause of death in the U.S. Numerous treatments for this disease have been proposed over the last two decades primarily aimed at reducing risk factors and correcting levels of abnormal lipids. Much less attention has been paid to reducing inflammation in spite of the fact that the majority of subjects in clinical trials to reduce atherosclerosis continue to have cardiovascular events in spite of aggressive LDL lowering therapy. These recurrent events are called residual cardiovascular risk and attempts to reduce it have not been successful. Understanding the pathogenesis of cardiovascular disease is critical to ultimately preventing and/or reversing its devastating effects in the U.S. population.

Atherosclerosis is an inflammatory, progressive disease that begins during fetal development and progresses throughout life [1]. As such, age is one of the primary determinants of cardiovascular risk [2]. The rate of development is increased by several well established risk factors including glucose intolerance, smoking, hypertension, and hyperlipidemia [3]. Early intervention into these factors has been recommended by many authors, and studies have demonstrated the benefits of this approach [4]. Based on these observations, atherosclerosis should be considered a chronic disease whose treatment will require a prolonged effort to reverse the atherosclerotic process.

Several therapeutic approaches have been successful in reducing and/or reversing the progression of atherosclerosis. All of these approaches have resulted in increasing the hepatic LDL receptor resulting in a reduction in circulating LDL cholesterol, including low cholesterol and saturated fat diets, ileal bypass surgery, intestinal cholesterol blockade by ezetimibe, hepatic cholesterol synthesis reduction by statins, and blockage of PCSK9 protein by monoclonal antibody inhibitors [5]. Even in individuals at low risk for ASCVD, the reduction of atherosclerotic cardiovascular events has been shown to be proportional to the reduction in circulating LDL cholesterol, with each mmol of reduction ( 48 $\mathrm{mg} / \mathrm{dl}$ ) resulting in a concurrent reduction in events of 11 per 1000 individuals within five years [6] [7]. To this end, several intravascular ultrasound studies 
have demonstrated that reversal of coronary atherosclerosis occurs when the LDL cholesterol approximates 65 to $70 \mathrm{mg} / \mathrm{dl}$ with statin therapy [8]. This process has been shown to begin in the carotid artery within one month of initiating statin therapy and continuing at one year and thereafter [9]. Stabilization (increase in thickness) of the atherosclerotic plaque's cap (the usual site of the plaque rupture into the coronary artery) can be shown to occur within nine months of initiating statin therapy [10].

Unfortunately, all of the pharmaceutical studies demonstrating a beneficial reduction in LDL concentration in reducing cardiovascular events have demonstrated a large remaining group of individuals with residual cardiovascular risk (Figure 1). This risk includes myocardial infarction, thrombotic stroke, acute severe angina, and peripheral vascular occlusion. This risk tends to decrease with time, presumably because with treatment unstable atherosclerotic plaques become stabilized against rupture into the coronary artery arterial lumen. However, during the first several years of initiating LDL lowering therapy, many patients continue to experience major cardiovascular events (residual risk).

\section{What Causes Residual Risk?}

Residual cardiovascular risk is primarily caused by the rupture of an unstable atherosclerotic plaque, with the resultant extrusion of thrombogenic material (including cholesterol, cholesterol esters, remnant lipoproteins, and lipoprotein [a]) into the arterial lumen [11]. The resultant thrombosis can result in partial or total obstruction of the coronary artery, resulting in unstable angina and myocardial infarction. Successful reduction of residual cardiovascular risk will require a rapidly acting therapeutic approach to stabilize atherosclerotic plaques with thin fibrous caps throughout the vascular tree. The thickness of the fibrous cap is a major determinant of the difference between an acute myocardial infarction and stable angina [12]. For example, in the infarct-related site of a study of

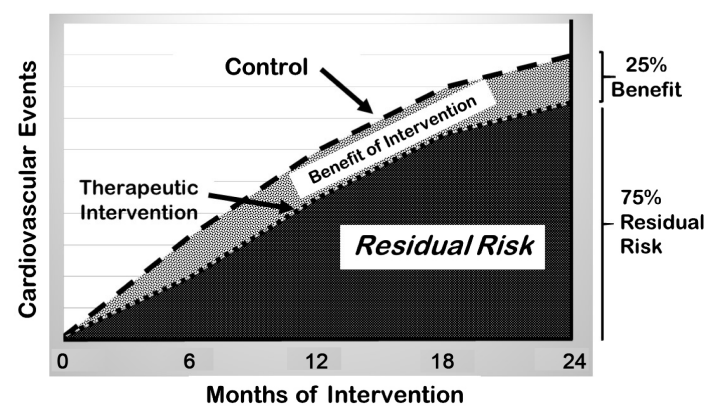

Figure 1. Time course of cardiovascular events during 24 months of therapy with a PCSK9 inhibitor compared to a statin control. In spite of a $15 \%$ reduction in events at one year and a $25 \%$ reduction at two years, many patients still had cardiovascular events in spite of dramatic lowering of LDL cholesterol with a PCSK9 inhibitor. The rate of residual risk cardiovascular events decreases with duration of the intervention. Note that the number of individuals having a cardiovascular event is significantly greater than the number of individuals in whom an event was prevented by the PCSK9 intervention. Data adapted from [16]. 
42 patients with acute myocardial infarction, the fibrous caps in their arteries averaged $57+/-12 \mu \mathrm{m}$ versus the fibrous caps instable angina patients of 180 $+/-65 \mu \mathrm{m}(p<0.001)$. In a pivotal study of plaque morphology from patients suffering from an acute myocardial infarction, Van der Wal and co-workers examined the culprit plaque lesion in 20 patients who died of a myocardial infarction [13]. They observed that the immediate site of plaque rupture was always associated with inflammation. They state that "inflammation plays a role in destabilizing the fibrous cap tissue and thus, in enhancing the risk of coronary thrombosis" (see Figure 2).

Regression of atherosclerosis occurs at an LDLc of approximately 65 to 70 $\mathrm{mg} / \mathrm{dl}$ [8] [14] [15]. However, this process requires a significant amount of time to become clinically significant (more than two years). Two recent publications emphasize this point. First, in the Fourier Clinical Trial, the PCSK9 inhibitor evolocumab was administered for 2 years and compared to a randomized placebo comparative group [16]. In spite of a significant reduction of CVD events in the PCSK9 group (15\% in year one, $25 \%$ in year two), this group still had a large number of residual events (1344 out of a study population of 13,784) during this time period. Second, using an alternative approach to lowering LDLc (or non HDL cholesterol) with the cholesterol ester transfer protein inhibitor anacetrapib, the REVEAL Clinical Trial reduced the CVD event rate $1 \%$ (10.8\% compared to $11.8 \%$ in the placebo group) [17]. However, no benefit of lowering the LDLc to approximately $30 \mathrm{mg} / \mathrm{dl}$ for the first two years was observed. To put this small benefit in proper perspective, out of 15,225 patients in the treated group, 1640 still had a cardiovascular event. Thus, although aggressive lowering of LDLc will reduce a small number of residual risk events, additional approaches will be necessary to further reduce the remaining heart attacks, strokes, and cardiovascular deaths.

The most likely additional factor to LDLc lowering is a significant reduction in inflammation. As shown in Figure 3, inflammation (as assessed with high

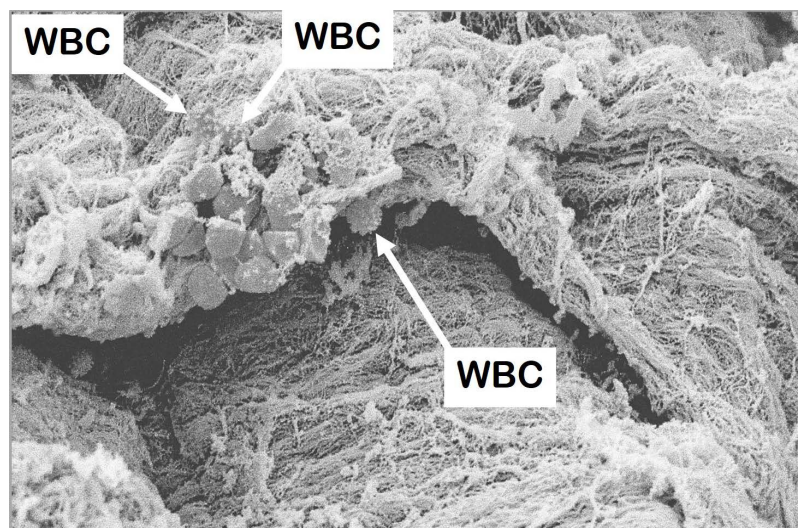

Figure 2. Electron micrograph of an atherosclerotic plaque showing entrapped red blood cells and attached lymphocytes (WBC). The stability of this cap is critical for preventing the rupture of the plaque into the arterial lumen. Local enzymes such as metalloproteinases degrade this cap whereas statins tend to stabilize it. Data adapted from [41]. 

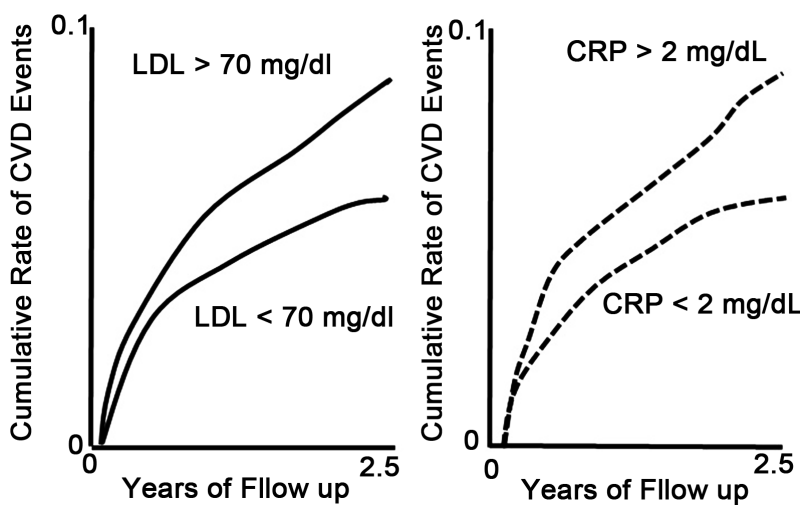

Figure 3. Relative importance of LDL versus inflammation (as assessed by CRP) in preventing CVD events after statin therapy. Note that inflammation is as important as hyperlipidemia in reducing cardiovascular risk. Data adapted from [26].

sensitivity C-reactive protein, i.e., hsCRP) is of equal importance to LDLc in reducing CVD events. It is likely that continued inflammation in atherosclerotic plaques leads to plaque rupture and arterial thrombosis. Although statins do reduce inflammation to some extent, this effect has not been sufficient to negate residual risk. The hypothesis that inflammation is critical to the pathogenesis of atherosclerotic events is supported by a recent publication demonstrating that the use of a specific monoclonal antibody that inhibits plaque inflammation (without altering plasma LDL concentration) reduced CVD events. Unfortunately, when the humanized monoclonal antibody canakinumab was used to decrease atherosclerotic plaque inflammation, an excess number of fatal infections were observed [18]. What is urgently needed is a specific inhibitor of plaque inflammation without significant adverse effects.

\section{Pathogenesis of Residual Risk}

Stabilization of an unstable atherosclerotic plaque is a complex process. Unstable plaques are characterized by thin fibrous caps, an excess of inflammatory cells, metalloproteinases, cytokines, a large lipid core, and minimal calcification [19] [20]. Critical to the stability of atherosclerotic plaque is the integrity of the fibrous cap that overlays the thrombogenic lipid core [21] (see Figure 2). This cap is formed from collagen secreted from smooth muscle cells. It may also contain inflammatory cells and cytokines. Enzymes secreted from macrophages and smooth muscle cells, termed metalloproteinases, may degrade the collagen and weaken the integrity of the fibrous cap. Thus, all plaques are in a precarious balance between increasing stability versus increasing cap dissolution. At the present time however, it is not clear which of these components are the most critical and need to be addressed first. Part of the beneficial effects of potent statin therapy has been attributed to its anti-inflammatory activity which may reduce the tendency of the plaque to rupture [22]. This activity may account for their very rapid benefit in patients with an acute coronary syndrome. In addition, statins may stabilize fibrous caps by increasing plaque calcification and re- 
duce the lipid core [23]. Unfortunately, these latter effects may take months to become effective in the majority of patients.

\section{What Do We Know about Atherosclerosis and Inflammation?}

The importance of inflammation in the pathogenesis of atherosclerosis was reviewed 18 years ago in a review entitled "Atherosclerosis-An Inflammatory Disease" [24]. In this review, many of the basic processes of the inflammatory process were detailed including the damage to the endothelial layer of the artery and its consequences. In addition, Ridker and coworkers have published several clinical studies which independently emphasizing the primary role of inflammation in pathogenesis of cardiovascular events [25]. They demonstrated that the beneficial effects of pravastatin could be attributed to a reduction in inflammation in individuals with normal cholesterol levels. Many changes in the artery affect the severity of the inflammatory response. For example, the flow of blood in the artery is altered by arterial branches, bifurcations, and acute curvatures that alter the flow of blood, resulting in a decrease in shear stress and an increase in turbulence [24]. At these sites, adhesion molecules form and attract monocytes and $\mathrm{T}$ lymphocytes which then migrate into the vessel wall.

In patients with an acute cardiovascular event treated with statins, it may be difficult to separate the beneficial effects of lowing LDL from the effects of reducing inflammation (i.e., CRP). However, this was done statistically by Ridkeret al. in 3745 subjects participating in the (PROVE IT-TIMI 22) study, a randomized controlled trial of patients experience an acute coronary syndrome [26]. These authors observed that for patients with post-treatment LDL cholesterol levels of more than $70 \mathrm{mg}$ per deciliter, the rates of recurrent events were 4.6 per 100 person-years among those with CRP levels of more than $2 \mathrm{mg}$ per liter and 3.2 events per 100 person-years among those with CRP levels of less than $2 \mathrm{mg}$ per liter. The respective rates among those individuals with LDL cholesterol levels of less than $70 \mathrm{mg}$ per deciliter were 3.1 and 2.4 events per 100 person-years $(P<0.001)$. These data strongly suggest that the level of CRP achieved with statin therapy has a major effect on the degree of benefit from lowering LDLc. Table 1 provides a risk assessment of future cardiovascular disease at various levels

Table 1. CRP risk estimate for cardiovascular disease in healthy men and women followed for 8 years.

\begin{tabular}{ccc}
\hline Quintile & CRP Range $\mathrm{mg} / \mathrm{L}$ & Risk Estimate \\
\hline $\mathbf{1}$ & $0.1-0.7$ & Low \\
$\mathbf{2}$ & $0.7-1.1$ & Mild \\
$\mathbf{3}$ & $1.2-1.9$ & Moderate \\
$\mathbf{4}$ & $2.0-3.8$ & High \\
$\mathbf{5}$ & $3.8-15.0$ & Highest \\
\hline
\end{tabular}

Data adapted from [27]. 
of CRP. This table is based on analysis of over 5000 men without known cardiovascular disease [27].

\section{Clinical Approach}

In the clinical trial literature, much emphasis has been placed on the benefit in reducing cardiovascular events with pharmaceutical intervention. Much less emphasis has been applied to the problem of residual risk, in spite of the fact that for every event prevented, three or more cardiovascular events have still occurred [28]. Although difficult to prove, it is likely that the residual risk events are caused by previously present unstable plaques rather than newly developed plaques (assuming that LDL cholesterol has been reduced below $65 \mathrm{mg} / \mathrm{dl}$ with high potency statins) [15].

What should be the physician's approach to residual risk? The first approach should be the acknowledgement that the danger from a recurrent event far exceeds any benefit of routine antilipidemic therapy [29]. The second approach should be the continued assessment of inflammation (i.e., by hsCRP analysis) at every visit in addition to monitoring a lipid profile The third approach should include the concept that atherosclerosis is a chronic disease which begins in childhood and progresses through decades of life and its reversal may require extended periods of time (years).

\section{Treatments}

Three pharmaceutical agents that have been demonstrated to: 1) reduce LDLc levels, 2) increase the hepatic LDL receptor concentration, and 3) reduce cardiovascular events are ezetimibe, high potency statins, and PCSK9 inhibitors [30] [31] [32]. The first two classes of drugs are now generic and should be used together to aggressively lower LDL cholesterol below $50 \mathrm{mg} / \mathrm{dl}$. The third class (PCSK9 inhibitors) is not only expensive but prescribing these agents is often restricted to specific internal medicine subspecialties. Statins and ezetimibe also decrease inflammation, an added benefit to their reduction of LDL cholesterol. Low dose aspirin $(81 \mathrm{mg} /$ day $)$ is also beneficial in reducing inflammation and is recommended in individuals with elevated CRP [33] [34]. In fact, the reduction in cardiovascular events with aspirin therapy has been attributed to its reduction in inflammation rather than its anti-platelet activity [35]. The importance of inflammation and its reduction with aspirin in preventing cardiovascular events was emphasized the Physicians Health Study, a randomized double blind placebo controlled study of 22,071 men followed for 8 years [35]. In this study, 543 men developed a heart attack or stroke. Their baseline CRP was $1.51 \mathrm{mg} / \mathrm{L}$, significantly greater than a comparable group of 543 men who did not develop cardiovascular disease. The use of aspirin in one fourth of the total study cohort significantly reduced cardiovascular events as well as reducing the level of CRP. Of particular interest was that aspirin was only effective in the top quartile of CRP in this study group, suggesting that elevated CRP was contributory to the 
cardiovascular event. The study was terminated early because of the highly significant reduction in cardiovascular disease induced with aspirin therapy.

Aggressive lifestyle changes that may involve reduction in sources of inflammation are also very important with normalization of blood pressure and hemoglobin A1C being paramount. A diet that achieves normal body weight that is low in saturated fat will also reduce the hepatic production of atherogenic lipids [36]. An exercise program is beneficial as several studies have documented a reduction in cardiovascular events when people who exercise are compared to people who do not [37] [38].

\section{Conclusion}

Reducing residual cardiovascular risk is a major challenge for all clinicians, primarily due to a lack of specific therapy to stabilize atherosclerotic plaques. There are no randomized controlled trials that specifically have addressed this issue. Statin therapy has been shown to reduce the immediate post-acute coronary morbidity and mortality, suggesting a beneficial effect [39]. A strong case can be made for also reducing inflammation [25]. In addition, antiplatelet therapy is often used to reduce the hypercoagulable state and is recommended in secondary prevention to reduce post cap rupture with arterial thrombosis [40]. However, the optimal approach to prevent atherosclerosis is by aggressively addressing cardiovascular risk factors, reducing LDL cholesterol below $50 \mathrm{mg} / \mathrm{dl}$, and reducing inflammation with a CRP less than $1.0 \mathrm{mg} / \mathrm{L}$. Additional research is urgently needed to address residual risk as a significant cause of morbidity and mortality.

\section{Funding}

There is no external funding support for this manuscript.

\section{Conflict of Interests}

The authors have no conflict of interests related to this manuscript.

\section{References}

[1] Schade, D.S., Cavanaugh, B., Ramo, B. and Eaton, R.P. (2016) The Application of the LDL Principle. World Journal of Cardiovascular Diseases, 6, 109-125. https://doi.org/10.4236/wjcd.2016.65012

[2] Tota-Maharaj, R., Blaha, M.J., McEvoy, J.W., Blumenthal, R.S., Muse, E.D., Budoff, M.J., et al. (2012) Coronary Artery Calcium for the Prediction of Mortality in Young Adults $<45$ Years Old and Elderly Adults $>75$ Years Old. European Heart Journal, 33, 2955-2962. https://doi.org/10.1093/eurheartj/ehs230

[3] Greenland, P., Knoll, M.D., Stamler, J., Neaton, J.D., Dyer, A.R., Garside, D.B., et al. (2003) Major Risk Factors as Antecedents of Fatal and Nonfatal Coronary Heart Disease Events. JAMA, 290, 891-897. https://doi.org/10.1001/jama.290.7.891

[4] Nasir, K., Rubin, J., Blaha, M.J., Shaw, L.J., Blankstein, R., Rivera, J.J., et al. (2012) Interplay of Coronary Artery Calcification and Traditional Risk Factors for the Pre- 
diction of All-Cause Mortality in Asymptomatic Individuals. Circulation: Cardiovascular Imaging, 5, 467-473. https://doi.org/10.1161/CIRCIMAGING.111.964528

[5] Silverman, M.G., Ference, B.A., Im, K., Wiviott, S.D., Giugliano, R.P., Grundy, S.M., et al. (2016) Association between Lowering LDL-C and Cardiovascular Risk Reduction among Different Therapeutic Interventions: A Systematic Review and Meta-Analysis. JAMA, 316, 1289-1297. https://doi.org/10.1001/jama.2016.13985

[6] Cholesterol Treatment Trialists' (CTT) Collaboration. (2010) Efficacy and Safety of More Intensive Lowering of LDL Cholesterol: A Meta-Analysis of Data from 170,000 Participants in 26 Randomised Trials. Lancet, 376, 1670-1681. https://doi.org/10.1016/S0140-6736(10)61350-5

[7] Cholesterol Treatment Trialists' (CTT) Collaborators, Mihaylova, B., Emberson, J., Blackwell, L., Keech, A., Simes, J., et al. (2012) The Effects of Lowering LDL Cholesterol with Statin Therapy in People at Low Risk of Vascular Disease: Meta-Analysis of Individual Data from 27 Randomised Trials. Lancet, 380, 581-590. https://doi.org/10.1016/S0140-6736(12)60367-5

[8] Gao, W.Q., Feng, Q.Z., Li, Y.F., Li, Y.X., Huang, Y., Chen, Y.M., et al. (2014) Systematic Study of the Effects of Lowering Low-Density Lipoprotein-Cholesterol on Regression of Coronary Atherosclerotic Plaques Using Intravascular Ultrasound. BMC Cardiovascular Disorders, 14, 60-78. https://doi.org/10.1186/1471-2261-14-60

[9] Nakamura, T., Obata, J.E., Kitta, Y., Takano, H., Kobayashi, T., Fujioka, D., et al. (2008) Rapid Stabilization of Vulnerable Carotid Plaque within 1 Month of Pitavastatin Treatment in Patients with Acute Coronary Syndrome. Journal of Cardiovascular Pharmacology, 51, 365-371. https://doi.org/10.1097/FJC.0b013e318165dcad

[10] Takarada, S., Imanishi, T., Kubo, T., Tanimoto, T., Kitabata, H., Nakamura, N., et al. (2009) Effect of Statin Therapy on Coronary Fibrous-Cap Thickness in Patients with Acute Coronary Syndrome: Assessment by Optical Coherence Tomography Study. Atherosclerosis, 202, 491-497.

https://doi.org/10.1016/j.atherosclerosis.2008.05.014

[11] Libby, P. and Theroux, P. (2005) Pathophysiology of Coronary Artery Disease. Circulation, 111, 3481-3488. https://doi.org/10.1161/CIRCULATIONAHA.105.537878

[12] Kubo, T., Imanishi, T., Takarada, S., Kuroi, A., Ueno, S., Yamano, T., et al. (2007) Assessment of Culprit Lesion Morphology in Acute Myocardial Infarction: Ability of Optical Coherence Tomography Compared with Intravascular Ultrasound and Coronary Angioscopy. Journal of the American College of Cardiology, 50, 933-939. https://doi.org/10.1016/j.jacc.2007.04.082

[13] van der Wal, A.C., Becker, A.E., van der Loos, C.M. and Das, P.K. (1994) Site of Intimal Rupture or Erosion of Thrombosed Coronary Atherosclerotic Plaques Is Characterized by an Inflammatory Process Irrespective of the Dominant Plaque Morphology. Circulation, 89, 36-44. https://doi.org/10.1161/01.CIR.89.1.36

[14] Nicholls, S.J., Ballantyne, C.M., Barter, P.J., Chapman, J., Erbel, R.M., Libby, P., et al. (2011) Effect of Two Intensive Statin Regimens on Progression of Coronary Disease. The New England Journal of Medicine, 365, 2078-2087. https://doi.org/10.1056/NEJMoa1110874

[15] Nissen, S.E., Nicholls, S.J., Sipahi, I., Libby, P., Raichlen, J.S., Ballantyne, C.M., et al. (2006) Effect of Very High-Intensity Statin Therapy on Regression of Coronary Atherosclerosis-The ASTEROID Trial. JAMA, 295, 1556-1565. https://doi.org/10.1001/jama.295.13.jpc60002

[16] Sabatine, M.S., Giugliano, R.P., Keech, A.C., Honarpour, N., Wiviott, S.D., Murphy, S.A., et al. (2017) Evolocumab and Clinical Outcomes in Patients with Cardiovas- 
cular Disease. The New England Journal of Medicine, 376, 1713-1722. https://doi.org/10.1056/NEJMoa1615664

[17] The HPS3/TIMI55-REVEAL Collaborative Group (2017) Effects of Anacetrapib in Patients with Atherosclerotic Vascular Disease. The New England Journal of Medicine, 377, 1217-1227. https://doi.org/10.1056/NEJMoa1706444

[18] Ridker, P.M., Everett, B.M., Thuren, T., MacFadyen, J.G., Chang, W.H., Ballantyne, C., et al. (2017) Antiinflammatory Therapy with Canakinumab for Atherosclerotic Disease. The New England Journal of Medicine, 377, 1119-1131. https://doi.org/10.1056/NEJMoa1707914

[19] Davies, M.J., Richardson, P.D., Woolf, N., Katz, D.R. and Mann, J. (1993) Risk of Thrombosis in Human Atherosclerotic Plaques: Role of Extracellular Lipid, Macrophage, and Smooth Muscle Cell Content. British Heart Journal, 69, 377-381. https://doi.org/10.1136/hrt.69.5.377

[20] Leber, A.W., Knez, A., White, C.W., Becker, A., von Ziegler, F., Muehling, O., et al. (2003) Composition of Coronary Atherosclerotic Plaques in Patients with Acute Myocardial Infarction and Stable Angina Pectoris Determined by Contrast-Enhanced Multislice Computed Tomography. American Journal of Cardiology, 91, 714-718. https://doi.org/10.1016/S0002-9149(02)03411-2

[21] Hansson, G.K. (2005) Inflammation, Atherosclerosis, and Coronary Artery Disease. The New England Journal of Medicine, 352, 1685-1695. https://doi.org/10.1056/NEJMra043430

[22] Libby, P. (2002) Inflammation in Atherosclerosis. Nature, 420, 868-874. https://doi.org/10.1038/nature01323

[23] Puri, R., Nicholls, S.J., Shao, M., Kataoka, Y., Uno, K., Kapadia, S.R., et al. (2015) Impact of Statins on Serial Coronary Calcification during Atheroma Progression and Regression. Journal of the American College of Cardiology, 65, 1273-1282. https://doi.org/10.1016/j.jacc.2015.01.036

[24] Ross, R. (1999) Atherosclerosis-An Inflammatory Disease. The New England Journal of Medicine, 340, 115-126. https://doi.org/10.1056/NEJM199901143400207

[25] Ridker, P.M., Rifai, N., Pfeffer, M.A., Sacks, F.M., Moye, L.A., Goldman, S., et al. (1998) Inflammation, Pravastatin, and the Risk of Coronary Events after Myocardial Infarction in Patients with Average Cholesterol Levels. Circulation, 98, 839-844. https://doi.org/10.1161/01.CIR.98.9.839

[26] Ridker, P.M., Cannon, C.P., Morrow, D., Rifai, N., Rose, L.M., McCabe, C.H., et al. (2005) C-Reactive Protein Levels and Outcomes after Statin Therapy. The New England Journal of Medicine, 352, 20-28. https://doi.org/10.1056/NEJMoa042378

[27] Ridker, P.M. (2001) High-Sensitivity C-Reactive Protein: Potential Adjunct for Global Risk Assessment in the Primary Preventon of Cardiovascular Disease. Circulation, 103, 1813-1818. https://doi.org/10.1161/01.CIR.103.13.1813

[28] Davidson, M.H. (2005) Reducing Residual Risk for Patients on Statin Therapy: The Potential Role of Combination Therapy. Davidson Reducing Residual Risk for Patients on Statin Therapy. American Journal of Cardiology, 96, 3K-13K. https://doi.org/10.1016/j.amjcard.2005.08.002

[29] Baigent, C., Keech, A., Kearney, P.M., Blackwell, L., Buck, G., Pollicino, C., et al. (2005) Efficacy and Safety of Cholesterol-Lowering Treatment: Prospective Meta-Analysis of Data from 90,056 Participants in 14 Randomised Trials of Statins. The Lancet, 366, 1267-1278. https://doi.org/10.1016/S0140-6736(05)67394-1

[30] Cannon, C.P., Blazing, M.A., Giugliano, R.P., McCagg, A., White, J.A., Theroux, P., et al. (2015) Ezetimibe Added to Statin Therapy after Acute Coronary Syndromes. 
The New England Journal of Medicine, 372, 2387-2397. https://doi.org/10.1056/NEJMoa1410489

[31] Ray, K.K., Cannon, C.P., McCabe, C.H., Cairns, R., Tonkin, A.M., Sacks, F.M., et al. (2005) Early and Late Benefits of High-Dose Atorvastatin in Patients with Acute Coronary Syndromes: Results from the PROVE IT-TIMI 22 Trial. Journal of the American College of Cardiology, 46, 1405-1410.

https://doi.org/10.1016/j.jacc.2005.03.077

[32] Everett, B.M., Smith, R.J. and Hiatt, W.R. (2015) Reducing LDL with PCSK9 Inhibitors-The Clinical Benefit of Lipid Drugs. The New England Journal of Medicine, 373, 1588-1591. https://doi.org/10.1056/NEJMp1508120

[33] Taylor, A.J., Bindeman, J., Feuerstein, I., Le, T., Bauer, K., Byrd, C., et al. (2008) Community-Based Provision of Statin and Aspirin after the Detection of Coronary Artery Calcium within a Community-Based Screening Cohort. Journal of the American College of Cardiology, 51, 1337-1341.

https://doi.org/10.1016/j.jacc.2007.11.069

[34] Campbell, C.L., Smyth, S., Montalescot, G. and Steinhubl, S.R. (2007) Aspirin Dose for the Prevention of Cardiovascular Disease: A Systematic Review. JAMA, 297, 2018-2024. https://doi.org/10.1001/jama.297.18.2018

[35] Ridker, P.M., Cushman, M., Stampfer, M.J., Tracy, R.P. and Hennekens, C. (1997) Inflammation, Aspirin, and the Risk of Cardiovascular Disease in Apparently Healthy Men. The New England Journal of Medicine, 336, 973-979. https://doi.org/10.1056/NEJM199704033361401

[36] Hu, F.B. and Willett, W.C. (2002) Optimal Diets for Prevention of Coronary Heart Disease. JAMA, 288, 2569-2578. https://doi.org/10.1001/jama.288.20.2569

[37] Blair, S.N., Kohl, H.W. III, Barlow, C.E., Paffenbarger Jr., R.S., Gibbons, L.W. and Macera, C.A. (1995) Changes in Physical Fitness and All-Cause Mortality: A Prospective Study of Healthy and Unhealthy Men. JAMA, 273, 1093-1098. https://doi.org/10.1001/jama.1995.03520380029031

[38] Myers, J., Prakash, M., Froelicher, V., Do, D., Partington, S. and Atwood, J.E. (2002) Exercise Capacity and Mortality among Men Referred for Exercise Testing. The New England Journal of Medicine, 346, 793-801. https://doi.org/10.1056/NEJMoa011858

[39] Schwartz, G.G., Olsson, A.G., Ezekowitz, M.D., Ganz, P., Oliver, M.F., Waters, D., et al. (2001) Effects of Atorvastatin on Early Recurrent Ischemic Events in Acute Coronary Syndromes-The MIRACL Study: A Randomized Controlled Trial. $J A M A, 285,1711-1718$. https://doi.org/10.1001/jama.285.13.1711

[40] Mehta, S.R. (2009) Aspirin for Prevention and Treatment of Cardiovascular Disease. Annals of Internal Medicine, 150, 414-416. https://doi.org/10.7326/0003-4819-150-6-200903170-00011

[41] Couzin-Frankel, J. (2016) Lipid Biology. Why High Good Cholesterol Can Be Bad News. Science, 351, 1126. https://doi.org/10.1126/science.351.6278.1126 